\title{
The Immunodepressive Effect of a Murine Plasmodium and its Interaction with Murine Oncogenic Viruses
}

\author{
By M. H. SALAMAN, NINA WEDDERBURN \\ Royal College of Surgeons, Lincoln's Inn Fields, London W.C.2 \\ AND L. J. BRUCE-CHWATT \\ London School of Hygiene and Tropical Medicine, Keppel Street, London W.C.I
}

(Accepted for publication 17 September 1969)

SUMMARY

Plasmodium berghei yoelii (p.b.y.) was found to cause an acute selflimiting infection in Balb/c mice, lasting for 14 to 18 days. A sharp fall in the primary response to sheep erythrocytes, as measured by the number of haemolytic plaque-forming cells in the spleen, and by the appearance of antibodies in the serum, coincided with high levels of parasitaemia between the 8th and II th days of p.b.y. infection. A secondary response to sheep erythrocytes was similarly affected when animals were infected with p.b.y. 9 days before the second antigen injection. Mice were resistant to reinfection with p.b.y., which produced either transient or no parasitaemia, and no immunodepression.

In mice carrying an immunodepressive leukaemogenic virus by vertical transmission, infection with a similar dose of p.b.y. was usually fatal.

Murine sarcoma virus (Harvey: m.s.v./H) produces tumours and splenomegaly in newborn mice but very rarely in adults. When injected into adult $\mathrm{Balb} / \mathrm{c}$ mice at the height of p.b.y. infection, m.s.v./H produced a high incidence of splenomegaly 4 weeks later, although the splenomegaly induced by the plasmodium alone had by then subsided.

These results are discussed in relation to Burkitt's (1969) hypothesis of a causal connection between chronic malarial infection and development of Burkitt lymphoma in children.

\section{INTRODUCTION}

The peculiar pattern of incidence of African lymphoma of childhood, first described by Burkitt (1959) and frequently confirmed (e.g. Haddow, 1963; Edington \& Maclean, 1964; Ten Seldam, Cooke \& Atkinson, 1966), has led to an intensive search for an insect-vectored virus as its cause. Although several viruses have been found associated with Burkitt's lymphoma (B.1.), and its distribution has been shown to correspond to that of certain mosquitoes, neither the hypothesis of viral causation, nor that of insect transmission, has been proved or disproved (see Wright, I967; Harris, 1967; Henle, 1968, for reviews).

Recently it has been suggested that the significance of B.l. distribution may not be its correspondence with the habitat of certain vectors, but with the prevalence of another vectored disease, malaria. O'Conor (196I) considered the possibility that parasitic infection might be a factor in the development of B.1., as had others for other lymphomas (Higginson, 1963). It was Dalldorf and his colleagues (1964) who first 
noted that high incidence of B.l. occurred in areas of holo-endemic malaria, i.e. where splenomegaly and parasitaemia are found in over $75 \%$ of children of 2 to 9 years, adult splenomegaly rate is low, and tolerance of infection in adults high. The association was found both in Kenya and in New Guinea. They suggested that the "primary cause' of B.l. is a widely distributed agent, but that lymphoma develops only when the reticuloendothelial system (r.e.s.) is stimulated by chronic malarial infection. Since then others have noted the correspondence between B.l. and malarial incidence (Edington \& Maclean, 1964; Henle, 1968). Burkitt (1969) has recently collected evidence for the association, and speculated upon its meaning.

Significant correlations are notoriously difficult to establish where standards of diagnosis and record differ widely, and where many variables cannot be controlled. Further surveys will be awaited with interest, but in the meantime it is worth considering how chronic malarial infection might influence the development of a lymphoma.

The authors quoted confine themselves to supposing, with Dalldorf and his colleagues (1964), that the r.e.s. may react 'differently' to the 'cause' of B.l. (presumably a virus) after malarial infection. What kind of difference might this be?

There are several possibilities, e.g. (i) malaria causes great hyperplasia of the r.e.s., and this may provide more cells able to support proliferation of an oncogenic virus, and thus increase the likelihood of malignant transformation; (ii) prolonged stimulation by plasmodial antigens may eventually lead to malignant change among immunereacting cells; or (iii) the parasite may produce an altered immune reactivity, leading to an abnormal response to an oncogenic virus or to the cellular antigens which it induces. For the first there is no factual evidence. The second has been suggested in other cases (Metcalf, I96I; Schwartz \& Beldotti, 1965; Walford, 1966; East \& de Sousa, 1966) but certainly not proved. The third is considered in the present work.

The strong cellular antigenicity of many strains of B.l. (Henle \& Henle, I966; O'Conor, I96I), the unusually high cure rate after relatively small doses of drugs (Ziegler et al. 1967; Burkitt, 1967), and the occurrence of spontaneous cures (D. P. Burkitt, personal communication), all suggest that the disease is strongly influenced by the patient's immune reaction. Some have postulated that the difference between B.1. and acute leukaemia is due to a difference in immune reaction to a causative agent (Burchenal, 1966; Clifford, 1966). Leukaemia, it is supposed, may follow an early (prenatal or neonatal) transmission of an oncogenic agent which induces tolerance, while B.l. may result from a later transmission (by contact or vector) of the agent which induces an immune reaction. Alternatively, a higher immune reactivity in the African B.1. patient may be due to intense exposure to a variety of other infectious agents (Henle \& Henle, I966), such as several species of plasmodia. In both cases the assumption is that the B.l. patient is making a stronger immunological response than the leukaemic to an oncogenic virus or to the cellular antigens which it induces.

If both conditions were caused by the same virus, antigenic cross-reactivity between the cells of B.l. and of leukaemias would be expected; none has been found so far (Henle \& Henle, 1966). If B.1. were merely leukaemia confined to certain sites by a strong immune response, we might expect it to arise in the presumptive sites of origin of leukaemic cells: lymphoid organs or bone marrow. B.l., at any rate in its early stages, conspiciously spares these sites. Moreover there is no evidence that B.1. patients react particularly strongly to other antigenic stimuli.

Although immunoglobulins IgM and IgG increase earlier in life and reach a higher 
adult level in Africans than in black or white residents in other countries, there is no evidence that the former have more active immune responses. In malarial and other parasitic diseases raised IgM and IgG levels are common, though only partially accounted for by a parallel increase of protective antibody against the causative organism (Smithers, 1967; Curtain, Kidson, Champness \& Gorman, 1964).

There is much evidence that procedures or agents which damage or functionally exhaust the r.e.s. affect the course of malarial and other parasitic infections (Goble \& Singer, 1960). Surprisingly little is known about the response of a host infected with malaria to other antigens. The only relevant report we have found is that of McGregor \& Barr (1962), who found a higher incidence of non-reactors to tetanus toxoid among malarious than non-malarious children in the Gambia. Intercurrent infections in malaria have been little investigated, and we do not know whether bacteria or viruses evoke a greater or a lesser antibody response from a host with an acute or long-term malarial infection. The effect of protozoan infections on the general capacity of an organism to produce immune responses to other antigens has been little explored.

Depression of immune responses by certain viruses and bacteria is well established, and has been recently reviewed (Salaman, I969; Floersheim, 1969). In the present work, methods used in the study of immunodepression by murine leukaemogenic viruses (Salaman \& Wedderburn, 1966; Wedderburn \& Salaman, 1968) have been employed to examine the effect of a murine plasmodium on immune responses in mice.

\section{METHODS}

Mice

Balb/c mice, maintained by brother-sister mating, were fed a pellet diet (FFG, made by F. G. Dixon Ltd., Ware, Herts) and water ad lib.

\section{Virus preparations}

Murine Sarcoma Virus (Harvey) (m.s.v./H). A 10 \% extract of livers and spleens from animals infected with this agent was received from Dr J. J. Harvey. It produced splenomegaly and multiple sarcomas in $100 \%$ of animals injected when newborn.

Urethane Leukaemia Virus (u.l.). This leukaemogenic virus was isolated by one of us from urethane-induced leukaemia (Salaman, 1963), and was found to pass by vertical transmission (v.t.). Balb/c mice carrying the virus (u.l./v.t.) develop gross splenomegaly with or without lymph node enlargement at 6 to 9 months.

\section{Plasmodial infection in mice}

In preliminary experiments several murine plasmodia were tested with the object of finding one which would produce, in mice of a strain previously used for immunological tests, an acute self-limiting disease of sufficient duration for the performance of these tests. The infection produced in 5- to 14 -week-old Balb/c mice by intraperitoneal (i.p.) injection of approximately $10^{6}$ organisms of $P$. berghei yoelii (p.b.y.) was found to be suitable.

A group of mice were injected with 70,000 infective sporozoites per mouse. Eight days later the mice, which had ro to $15 \%$ parasitized erythrocytes were bled, and the blood, diluted I I I with Ringer containing I $5 \%$ glycerin and 20 units heparin/ ml., was stored at $-70^{\circ}$. For experimental purposes two mice received i.p. $0.1 \mathrm{ml}$. of 
the stored infected blood. Six days later they were bled, and the blood was diluted so that $10^{6}$ parasitized erythrocytes were contained in a volume suitable for i.p. injection into test mice.

In the infection caused by this dose, parasitaemia, first detectable on the 3rd day, rose, sometimes showing a small peak on the 4 th day, until on the 9 th to IIth day mice had $\mathrm{I} 5$ to $35 \%$ parasitized erythrocytes The percentage then declined until the 15 th to 17 th day, when less than I \% parasitized erythrocytes were found. Splenomegaly, detectable by palpation on the 3 rd day, reached a peak about the time of the peak of parasitaemia, when spleens weighed I to $2 \mathrm{~g}$. (the normal weight for adult mice of this strain is 0.1 to $0.15 \mathrm{~g}$.), and persisted for some time after parasitaemia was nolonger detectable. The mice looked fluffed and hunched and were anaemic (haematocrit 25 to $35 \%$ ) between the 8 th and 13 th days, and then recovered. No deaths occurred among mice aged five weeks or more, but of a batch of three-week-old mice which received a smaller dose ( $10^{5}$ parasitized erythrocytes) half died.

\section{Immunological tests}

Serum haemagglutinin (ha) and haemolysin (hl) titres and also the number of haemolytic plaque-forming cells (p.f.c.) in the spleen were determined after the intravenous (i.v.) injection of $2.5 \times 10^{8}$ sheep erythrocytes. The number of p.f.c. were determined by the method of Jerne, Nordin \& Henry (1963) with minor modifications. Details of the immunological tests used have been described elsewhere (Salaman \& Wedderburn, I966; Wedderburn \& Salaman, 1968).

\section{RESULTS}

\section{Immune response to sheep erythrocytes in mice infected with malaria}

Eight-week-old Balb/c mice were infected with plasmodia, and equal groups were then given $2.5 \times 10^{8}$ sheep erythrocytes (s.e.) i.v. 4,7 and 9 days later. Seven days after antigen injection ha and hl titres of the first two groups showed a three- to fourfold depression compared with uninfected controls; however, the titres then began to rise, and had reached control levels 3 weeks after s.e. injection. Animals which received s.e. 9 days after p.b.y. had no detectable ha or hl 7 days later, and antibody was still barely detectable 3 weeks after antigen injection.

In unimmunized Balb/c mice there are 40 to I 40 p.f.c. per spleen against s.e. In mice infected with p.b.y. a gradual rise in this 'background' number of p.f.c. began 4 days after infection, and reached 500 to 1000 p.f.c. by the 7 th day. The count remained at about this level till the I3th day, after which it declined slowly, but was still significantly raised 2 months after infection.

The number of p.f.c. per spleen 4 days after a dose of $2.5 \times 10^{8}$ s.e. was then determined for groups of four mice which had received the antigen at different intervals after infection with the plasmodium. The results are shown in Fig. I, together with the degree of parasitaemia at the time of s.e. injection. Notwithstanding the rise in 'background' p.f.c. in infected mice there was a short but severe impairment of the immune response to the antigen when given during a period which roughly coincided with the period of maximum parasitaemia.

The number of p.f.c. per spleen in mice which had received s.e. Io days after p.b.y. was followed for some time, to ascertain whether the reduction in the p.f.c. response at 
4 days was merely a delay. Results showed that there was no significant delay, but a true reduction. The number of p.f.c. in immunized infected mice never rose higher than I600 per spleen. Uninfected immunized mice, on the other hand, showed a peak response of 250,000 to 300,000 p.f.c. per spleen on day 4 .

The primary p.f.c. response after repeated injections of plasmodia was next examined. When mice received a second injection of p.b.y., or a series of four injections at fortnightly intervals, no recurrence of parasitaemia was observed, and when a second injection of p.b.y. was followed by s.e. there was no depression of the p.f.c. response.

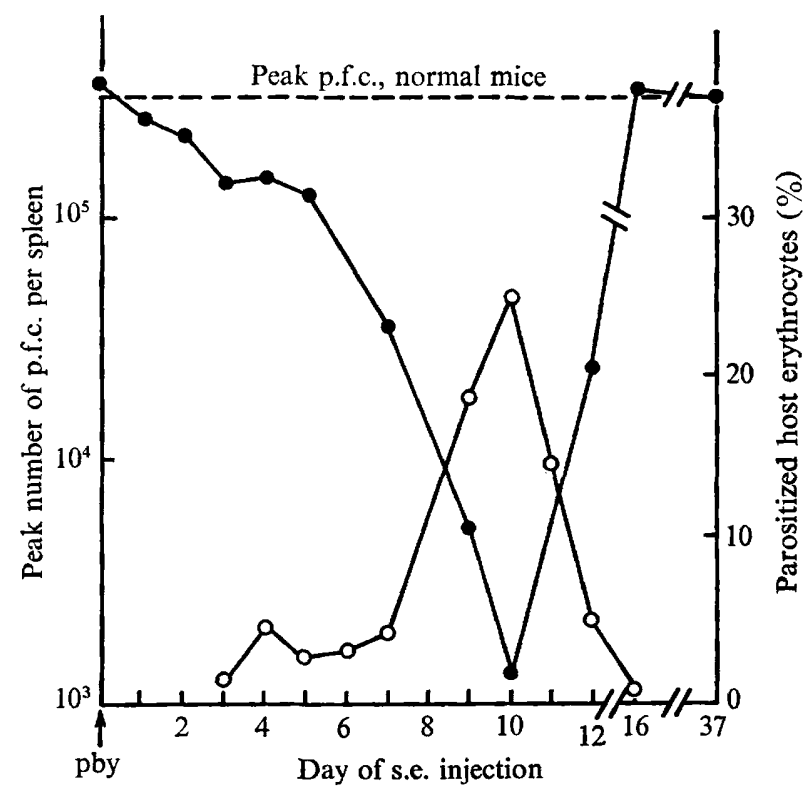

Fig. I. Effect of Plasmodium berghei yoelii (p.b.y.), injected at various times before a standard dose of sheep erythrocytes (s.e.) on the peak number of haemolytic plaque-forming cells (p.f.c.) per spleen. Four mice in each group. Mice received $10^{6}$ parasitized erythrocytes (p.b.y.) intraperitoneally. $2.5 \times 10^{8}$ sheep erythrocytes were injected i.v. on the day indicated. Mean peak number of p.f.c. per spleen (measured 4 days after s.e. injection): - - mean percentage of erythrocytes parasitized: $-\mathrm{O}-\mathrm{O}-$.

The effect of plasmodial infection on the secondary response was examined briefly. When mice were infected 9 days before a primary injection of s.e., to which they showed an extremely depressed response, as described above, they nevertheless responded vigorously to a second dose of s.e. given 5 weeks after the first: they were obviously not tolerant to the antigen. However, further work is necessary to determine whether this response has the characteristics of a primary or of a secondary reaction. On the other hand mice which received p.b.y. 9 days before the second of two injections of s.e. separated by 4 to 5 weeks showed a ro-fold rise in the residual p.f.c. count usually found 4 to 5 weeks after a primary s.e. injection and by a 20-fold depression of the response to the second s.e. injection. This result is qualitatively similar to that observed when p.b.y. was given before a primary s.e. injection. 


\section{Effect of malarial infection on the pathogenicity of an oncogenic virus}

Since murine plasmodial infection depressed the immune response to s.e., it was of interest to discover whether it altered the response to infection by oncogenic viruses. Murine sarcoma virus (Harvey) (m.s.v./H) (Harvey, 1964) was chosen as an example, because it causes tumours, splenomegaly, and early death when injected into newborn animals, but is much less pathogenic in adults. If the reason for this difference, in this case and in those of other viruses with similar age-dependent pathogenicity, were the relative incompetence of the neonatal immune system, then it would be expected that the effects of m.s.v./H in plasmodium-infected adults would approximate to those in normal newborn mice.

Two groups of eight Balb/c mice aged 10 weeks received $10^{6}$ p.b.y. per mouse. Ten days later one group received i.p. $0.1 \mathrm{ml}$. of m.s.v.-infected plasma and a third untreated group also received m.s.v.

Four and a half weeks after the injection of m.s.v. the mice were killed. The group which had received p.b.y. followed by m.s.v. had spleens weighing from 0.25 to $1.5 \mathrm{~g}$. (average $0.7 \mathrm{I}$ g.), with numerous superficial nodules. Histologically they presented the usual appearance of m.s.v. spleens, but contained in addition a considerable amount of malarial pigment (F. C. Chesterman, personal communication). One mouse which had received m.s.v. only appeared sick at this time. It had a ruptured spleen weighing $0.47 \mathrm{~g}$., and was the only mouse in this group in which any evidence of m.s.v. infection was seen; the others all had spleens weighing less than $0.25 \mathrm{~g}$., and showed no gross pathology. No tumours were found in either of the m.s.v.-infected groups. Animals which had received p.b.y. only had spleens weighing less than $0.25 \mathrm{~g}$., containing much malarial pigment.

It is clear therefore that the pathological effects of m.s.v. in the spleens of adult mice were greatly increased by prior infection with p.b.y., while the production of solid tumours was not increased, at least over the time-span used.

\section{Malarial infection in mice carrying a vertically transmitted virus derived from urethane-induced leukaemia}

Balb/c mice carrying the virus u.l. by vertical transmission (u.l./v.t.) develop gross splenomegaly, with or without lymph node enlargement, at 6 to 9 months. Gradually increasing splenomegaly, and a slight degree of immune depression, are found before this, i.e. from about the 8th week. U.1./v.t. mice of 6 to 10 weeks of age were infected with p.b.y. in the usual way, in the hope that recovered mice might show a changed incidence or time of development of overt leukaemia.

The malarial infection progressed in the usual way until the 12th day. After that, instead of recovering like normal Balb/c, the u.l./v.t. mice continued to deteriorate, and out of 19 mice 16 died between the 15 th and 2 Ist days, with $50 \%$ or more parasitized erythrocytes and gross anaemia.

The severe but short-lived immune depression which occurs during a primary infection of Balb/c mice with p.b.y. does not prevent the development of resistance which terminates the infection and prevents reinfection. However, the above experiment shows that development of effective resistance is prevented by the presence of a virus which exerts a more prolonged immunodepressive effect than the parasite itself. 


\section{DISCUSSION}

The question originally asked, whether malarial infection can predispose to infection by an oncogenic virus, has not been fully answered. It has been shown that a murine plasmodium severely depresses immune reactivity for a short period at the height of parasitaemia, and that the pathogenicity of a murine sarcoma virus injected during that period is enhanced. Conversely it has been shown that mice carrying a leukaemogenic and immunodepressive virus succumb to a dose of Plasmodium berghei yoelli which is followed by only temporary illness in normal mice.

An incidental finding was that the number of spleen cells capable of lysing sheep erythrocytes in unimmunized mice is significantly raised in plasmodial infection. This recalls the suggestion that increased titres of haemolysins and haemagglutinins to foreign erythrocytes in Africans may be related to infectious processes, especially malaria (Zuckerman, I966; Kano, McGregor \& Milgrom, 1968).

There is little evidence to indicate the mechanism of immunodepression in this case. The infected mouse is carrying a large burden of foreign antigen at the time of maximum immune depression, and the possibility of antigenic competition is not excluded. This possibility, in relation to immune depression by a virus, was discussed in a previous paper, and suggestive though not conclusive evidence against it was presented (Wedderburn \& Salaman, 1968). Further work is needed to determine what part, if any, antigenic competition plays in immunodepression by plasmodial infection.

The best animal model of the situation which Burkitt has suggested in man would be chronic plasmodial infection in a mouse carrying a leukaemogenic virus. Our attempt to examine the immune status of mice chronically infected with the plasmodium has been frustrated by solid resistance to reinfection. Repeatedly injected mice show no illness and no immunodepression. However, Jerusalem (I968) repeatedly infected Swiss mice with $P$. berghei berghei, usually lethal in these mice, by cutting short the initial infection with a $p$-aminobenzoic acid-free diet. At I I days these mice failed to reject both homo- and allogeneic skin grafts, and after 6 months of repeated injections there was some evidence of increased incidence of malignant lymphoma (Jerusalem, 1968, and personal communication).

The significance of these findings in the relation of plasmodial infection to neoplasia is a matter for speculation until further evidence accumulates. A range of immune reactions, cell-associated as well as humoral, against a variety of antigens, should be examined at various stages of plasmodial infection.

The early immunodepression in acute plasmodial infection of mice, and the increased incidence of malignant lymphoma in chronically infected mice, suggests, by analogy, a reason for the correlation which Burkitt has noted between the incidence of Burkitt lymphoma and malaria in man.

In planning this work we received valuable advice from Professor P. C. Garnham. We are indebted to Dr F. C. Chesterman for histological diagnosis, to Mr R. S. Killick Kendrick, Mrs B. Adkins and Mr R. Saldanha for technical, and to Miss C. Cowen for secretarial, assistance. 


\section{REFERENCES}

BuRChENAL, J. H. (1966). Geographic chemotherapy-Burkitt's tumour as a stalking horse for leukemia. Cancer Res. 26, 2393.

BURKITT, D. P. (1959). A sarcoma involving the jaws in African children. Br. J. Surg. 46, 218.

BURKITT, D. P. (1967). Chemotherapy of jaw tumours. In Treatment of Burkitt's Tumour. UICC Monograph, vol. 8, p. 94. Berlin, Heidelberg, New York: Springer-Verlag.

BURKITT, D. P. (1969). Etiology of Burkitt's lymphoma-an alternative hypothesis to a vectored virus. J. natn. Cancer. Inst. 42, I9.

Clifford, P. (1966). Further studies on the treatment of Burkitt's lymphoma. E. Afr. med. J. 43, I79.

Curtain, C. C., Kidson, C., Champness, D. L. \& Gorman, J. G. (I964). Malaria antibody content of gamma ${ }_{2} \mathrm{~S}$ globulin in tropical populations. Nature, Lond. $203, \mathrm{I} 366$.

Dalldorf, G., Linsell, C. A., Barhart, F. E. \& Martyn, R. (I964). An epidemiological approach to the lymphomas of African children and Burkitt's sarcoma of the jaws. Perspect. Biol. Med. 7 , 435 .

EAST, J. \& DE SousA, M. A. B. (1966). The thymus autoimmunity and malignancy in New Zealand black mice. Natn. Cancer Inst. Monogr. 22, 605.

EDINGTON, G. M. \& MACLEAN, C. M. U. (1964). Incidence of the Burkitt Tumour in Ibadan, Western Nigeria. Br. med. J. I, 264.

Floersherm, G. L. (1969). Suppression of cellular immunity by Gram negative bacteria. Antibiotic. Chemother. 15, 407.

GoBLE, F. C. \& SINGER, I. (1960). The reticuloendothelial system in experimental malaria and trypanosomiasis. Ann. N.Y. Acad. Sci. 88/1, I49.

HADDow, A. J. (1963). An improved map for the study of Burkitt's lymphoma syndrome in Africa. E. Afr. med. J. 40, 429.

HARRIs, R. J. (1967). A virus aetiology for Burkitt's tumour. Int. J. Canc. 2, 559.

HARVEY, J. J. (1964). An unidentified virus which causes the rapid production of tumours in mice. Nature, Lond. 204, 1104.

HENLE, G. \& HENLE, W. (I966). Immunofluorescence in cells derived from Burkitt's lymphoma. $J$. Bact. 9I, 1248.

HENLE, W. (1968). Evidence for viruses in acute leukaemia and Burkitt's tumour. Cancer, N.Y. 2 r, 580.

Higginson, J. (1963). Geographical variations in observed frequencies of cancer of different sites. In Cancer Progress. Ed. by R. W. Raven, p. 80. London: Butterworth.

JERNE, N. K., NoRdin, A. A. \& HENRY, C. (1963). The agar plaque technique for recognising antibodyproducing cells. In Cell-bound Antibodies, Ed. by B. Amos and H. Koprowski, p. I09. Philadelphia: Wistar Inst. Press.

JeRUSALEM, C. (1968). Relationship between malaria infection (Plasmodium berghei) and malignant lymphoma in mice. $Z$. Tropenmed. Parasit. $19,94$.

Kano, K., McGregor, I. A. \& Milgrom, F. (I968). Haemagglutinins in sera of Africans of Gambia. Proc. Soc. exp. Biol. Med. I29, 849.

MCGREGOR, I. A. \& BARR, M. (I962). Antibody response to tetanus toxoid inoculation in malarious and non-malarious Gambian children. Trans. R. Soc. trop. Med. Hyg. 36, 364.

METCALF, D. (I96I). Reticular tumours in mice subjected to prolonged antigenic stimulation. $B r . J$. Cancer 15, 769 .

O'Conor, G. T. (1961). Malignant lymphoma in African children. II. A pathological entity. Cancer $N . Y, \mathbf{x 4}, 270$.

Salaman, M. H. (1963). Attempt to isolate a leukaemogenic virus from urethane-induced leukaemia in mice. Rep. Br. Emp. Cancer Campn 40, 220.

Salaman, M. H. (1969). Immunodepression by viruses. Antibiotic. Chemother. 15, 393.

Salaman, M. H. \& WedderbuRn, N. (1966). The immunodepressive effect of Friend virus. Immuno$\log y$ 10, 445 .

SCHWARTZ, R. S. \& BeLDotTI, L. (1965). Malignant lymphomas following allogenic disease: Transition from an immunological to a neoplastic disorder. Science, N.Y. 149, 1511 .

SMITHERS, S. R. (1967). The induction and nature of antibody response to parasites. In Immunological Aspects of Parasitic Infections. Ed. by O. Bier. Pan-Amer. Health Org. WHO Scientific Publ. r5o, p. 43. 
Ten Seldam, R. E. J., Cooke, R. \& Atkinson, L. (1966). Childhood lymphoma in the Territories of Papua and New Guinea. Cancer, N.Y. 19, 437.

WALFORD, R. L. (I966). Increased incidence of lymphoma after injections of mice with cells differing at weak histocompatibility loci. Science, N.Y. 152, 78 .

Wedderburn, N. \& Salaman, M. H. (1968). The immunodepressive effect of Friend virus. II. Reduction of splenic haemolysin-producing cells in primary and secondary responses. Immunology $\mathbf{1 5}$, 439.

Wright, D. H. (1967). The epidemiology of Burkitt's tumour. Cancer Res. 27, 2424.

Ziegler, L., Cohen, M. H., Vogel, C. L., Sheagren, J. N. \& Carbone, P. P. (1967). Immunologic studies in American patients with Burkitt-like lymphoma. Cancer Res. 27, 2527.

ZuCKERMAN, A. (1966). Recent studies on factors involved in malarial anaemia. Military Med. Suppl. I3I, I2OI. 